\title{
Preparo de Nanocompósitos de Poli(Succinato de Butileno) (PBS) e Argila Motmorilonita Organofílica via Polimerização In Situ
}

\author{
Letícia P. Ferreira, Andrei N. Moreira, Fernando G. de Souza Jr. \\ Instituto de Macromoléculas Professora Eloisa Mano - IMA, Universidade Federal do Rio de Janeiro - UFRJ \\ José Carlos Costa da Silva Pinto \\ Programa de Engenharia Química - COPPE, Universidade Federal do Rio de Janeiro - UFRJ
}

\begin{abstract}
Resumo: Nanocompósitos de poli(succinato de butileno) (PBS) e argila montmorilonita organofílica foram sintetizados via polimerização in situ, em três diferentes concentrações (4, 6 e $8 \%$ em massa). Os produtos obtidos foram caracterizados por diversas técnicas. Entre elas, a difração de raios $\mathrm{X}$ foi útil para comprovar o aumento do espaçamento interlamelar da argila devido à incorporação das cadeias de polímero. As técnicas de análise térmica mostraram que o método de polimerização in situ gerou materiais com menores estabilidades térmicas, comparadas ao polímero puro, devido à dificuldade de crescimento das cadeias de polímero na presença da argila. A técnica de RMN de baixo campo permitiu identificar que a estrutura esfoliada predomina nos nanocompósitos.
\end{abstract}

Palavras-chave: Poli(succinato de butileno), polímero biodegradável, argila organofilica, nanocompósito, polimerização in situ.

\section{Preparation of Nanocomposites Based on Poly(Butylene Succinate) and Montmorillonite Organoclay via In Situ Polymerization}

\begin{abstract}
Nanocomposites based on poly(butylene succinate) (PBS) and organophilic montmorillonite were synthesized via in situ polymerization using three different clay compositions $(4,6$ and $8 \mathrm{wt} \%)$. The products were characterized by several different techniques. X-ray diffraction was useful to confirm the increase of the interlayer spacing of the clay due to the presence of the polymer chains among layers. Thermal analysis indicated that the polymerization method chosen led to materials with lower thermal stability compared to the pure PBS, due to the difficulty of chain growth in the presence of the clay. Low-field NMR technique was used to assess clay dispersion in the polymer, with exfoliated structures predominating in the nanocomposites.
\end{abstract}

Keywords: Poly(butylene succinate), biodegradable polymer, organophilic clay nanocomposite, in situ polymerization.

\section{Introdução}

O desenvolvimento de materiais poliméricos biodegradáveis com boas propriedades tem se tornado o foco de pesquisas ao redor do mundo na tentativa de solucionar o problema do acúmulo de resíduos sólidos. Além disso, deseja-se reduzir a dependência de recursos fósseis para a fabricação de diversos produtos a partir do desenvolvimento de materiais poliméricos "verdes", que podem ser reciclados ou degradados em ambiente controlado $^{[1]}$.

Os poliésteres alifáticos têm sido considerados por muitos, como os materiais biodegradáveis mais competitivos do futuro, o que incentiva a pesquisa desse polímero por diversos grupos ${ }^{[2]}$. Estes poliésteres podem ser degradados na forma de $\mathrm{CO}_{2}$ e $\mathrm{H}_{2} \mathrm{O}$ pela ação de enzimas ou micro-organismos, em condições específicas de compostagem, porém são estáveis em atmosfera normal ${ }^{[3]}$. Entre eles, o PBS é um poliéster alifático que pode ser sintetizado quimicamente por meio da policondensação do 1,4-butanodiol com o ácido succínico, ambos biomonômeros de origem renovável, obtidos a partir da biomassa, apresentando, portanto, menores custos de produção em relação aos produtos similares provenientes do petróleo ${ }^{[4]}$. Além disso, o PBS possui propriedades vantajosas, como boas resistências térmica (estável até aproximadamente $200^{\circ} \mathrm{C}$ ) e química (estabilidade a solventes e outros produtos químicos), e ainda, uma excelente processabilidade ${ }^{[5]}$. Dessa forma, representa uma opção interessante a diversas aplicações, como na produção de materiais biomédicos, filmes agrícolas, embalagem, produtos espumados e até materiais para a indústria automobilística ${ }^{[6]}$.

Recentemente, o uso de argila em nanocompósitos poliméricos tem sido bastante explorado no âmbito acadêmico e industrial ${ }^{[7]}$. Isso se deve ao fato deste tipo de nanocompósito apresentar, em comparação aos compósitos convencionais, melhores propriedades físicas e/ou químicas com a adição de uma pequena quantidade de carga ${ }^{[8]}$. Essas melhorias são observadas em diversas

Autor para correspondência: Fernando G. de Souza Jr., Instituto de Macromoléculas Professora Eloisa Mano - IMA, Universidade Federal do Rio de Janeiro - UFRJ, Cidade Universitária, Av. Horácio Macedo, 2030, Centro de Tecnologia, Prédio do Bloco J, CEP 21941-598, Rio de Janeiro, RJ, Brasil, e-mail: fgsj@ufri.br 
propriedades, tais como o aumento do módulo de Young, o aumento da estabilidade térmica e a melhoria das propriedades de barreira a gases, entre outras ${ }^{[9,10]}$. As argilas organofílicas possuem baixo custo, bem como elevada razão de aspecto e a grande área específica $\left(\sim 800 \mathrm{~m}^{2} / \mathrm{g}\right)$, contribuindo para uma maior interação com o polímero; associada a resistência das partículas a solventes e a temperaturas elevadas, geralmente empregadas nas polimerizações que geram poliésteres ${ }^{[11]}$. Além disso, o fato da argila organofílica ter um maior espaçamento basal permite que o polímero possa ser intercalado entre as camadas, resultando no nanocompósito polímero / argila organofílica. A literatura reporta o preparo de nanocompósitos de PBS e argila principalmente por meios mecânicos, como a extrusão ${ }^{[12,13]}$. Por outro lado, a técnica de polimerização in situ, contribui para uma melhor dispersão da carga, pois o polímero é sintetizado em meio a esta ${ }^{[14]}$, permitindo a obtenção de estruturas esfoliadas, capazes de conferir melhores propriedades ao material ${ }^{[15]}$.

O principal objetivo deste trabalho foi o preparo de nanocompósitos de PBS e argila organofílica por meio da técnica de polimerização in situ. A polimerização foi feita em massa, o que, até onde conseguimos averiguar, é praticamente inexplorado para o polímero/compósito em questão. Os materiais obtidos foram caracterizados por diversas técnicas, entre elas a difração de raios $\mathrm{X}$ (DRX), a análise termogravimétrica (TGA), a calorimetria exploratória diferencial (DSC) e a ressonância magnética nuclear de baixo campo (RMN-BC). Os resultados de FTIR demonstram a obtenção do PBS a partir da observação de suas bandas características, bem como revelam a presença de quantidades crescentes de argila. Essa avaliação foi feita com o uso da técnica do erro médio quadrático, obtido por regressão linear via mínimos quadrados. Os valores obtidos por essa técnica foram confirmados por TGA. Já a técnica de DRX mostrou a obtenção de estruturas intercaladas nos compósitos, confirmada pela técnica de RMN-BC. Assim, além da natureza inédita do trabalho, esse artigo contribui para o avanço do conhecimento sobre o preparo de materiais nanocompósitos de matriz PBS, que, devido ao barateamento dos monômeros obtidos via rota biotecnológica, será um dos polímeros mais explorados economicamente em poucos anos.

\section{Experimental}

\section{Materiais}

Ácido Succínico P.A. ACS (99,0\%), 1,4 butanodiol P.S. (99,3\%), anidrido succínico P.A. (99,0\%), ácido sulfúrico P.A. (95-99\%) e glicerina (glicerol) P.A. ACS (99,5\%) foram fornecidos pela VETEC. O catalisador trióxido de antimônio $\left(\mathrm{Sb}_{2} \mathrm{O}_{3}\right)$ foi fornecido pela BRASKEM. Octoato de estanho ( SnOct $\left._{2}\right)(28 \%$ de estanho) foi fornecido pela MOMENTIVE. Tetrabutoxititânio $\left(\mathrm{Ti}(\mathrm{OBu})_{4}\right)$ foi fornecido pela SIGMA-ALDRICH. A argila montmorilonita (MMT) organofílica industrial, modificada por metil octadecil dihidroxietil amônio foi gentilmente doada pela Braskem. Todos os reagentes, bem como a argila, foram usados como recebidos.

\section{Síntese do PBS e dos nanocompósitos}

A síntese do polímero foi feita empregando a técnica de polimerização em massa. Foi utilizado um sistema fechado, a vácuo, com fluxo de nitrogênio para a síntese do polímero. O sistema foi agitado magneticamente, sob fluxo de nitrogênio com pressão de, aproximadamente, 0,75 bar. A mistura reacional continha uma quantidade equimolar $(0,304 \mathrm{~mol})$ de ácido succínico e 1,4-butanodiol. A temperatura foi ajustada para $150{ }^{\circ} \mathrm{C}$ e a reação teve duração de 5 horas. Após esta etapa, foi adicionado $0,3 \% \mathrm{p} / \mathrm{p}$ de catalisador tetrabutoxititânio $\left(\mathrm{Ti}(\mathrm{OBu})_{4}\right)$ no meio reacional e a temperatura foi ajustada para $200^{\circ} \mathrm{C}$, permanecendo assim por 12 horas, o que permitiu alcançar uma conversão de aproximadamente $99 \%$. A síntese do nanocompósito de PBS com a argila in situ procedeu de maneira similar à descrita para a síntese do PBS puro, porém com a adição da argila no início da reação junto à mistura de monômeros, antes mesmo do aquecimento.

Foram produzidos os compósitos com as concentrações teóricas de 4, 6 e $8 \%(\mathrm{p} / \mathrm{p})$ de argila montmorilonita organofílica (PBS-4, PBS-6, PBS-8).

\section{Caracterização}

Espectroscopia de infravermelho com transformada de Fourier usando acessório de reflexão total atenuada (FTIR-ATR): As amostras de polímero puro (PBS), de argila modificada e dos compósitos de PBS/argila foram analisados por FTIR-ATR. A análise foi feita no espectrômetro com transformada de Fourier PerkinElmer 1720X, no qual foi acoplado um acessório ATR com cristal de diamante. A faixa analisada foi de 4000 a $650 \mathrm{~cm}^{-1}$, com resolução de $4 \mathrm{~cm}^{-1}$ e em atmosfera inerte. As transmitâncias dos compósitos e do polímero puro foram comparadas estatisticamente. Para isso, os dados da análise foram normalizados em um intervalo de 1 a 100. Com auxílio de uma regressão linear via mínimos quadrados, foi possível obter os valores de correlação $\left(\mathrm{R}^{2}\right)$ e do erro médio quadrático (RMSE) da dispersão de dados gerada pela construção de gráficos comparativos entre os valores das transmitâncias (normalizadas entre 1 e 100) dos compósitos contendo a argila contra o PBS puro, que serviu como referência de material sem carga. Os detalhes do procedimento são mostrados em outro trabalho do grupo ${ }^{[16]}$.

\section{Análise termogravimétrica (TGA)}

As amostras do polímero e dos compósitos foram analisadas por termogravimetria pelo método de rampa de aquecimento em equipamento TA Instruments TGA Q500 - V6.7 Build 203, em atmosfera inerte de nitrogênio (gás de purga - $40 \mathrm{~mL} \cdot \mathrm{min}^{-1}$ ) e nas temperaturas entre $25^{\circ} \mathrm{C}$ e $700{ }^{\circ} \mathrm{C}$, com taxa de aquecimento de $20^{\circ} \mathrm{C} \cdot \mathrm{min}^{-1}$. Foi empregada uma isoterma de 3 minutos a $700{ }^{\circ} \mathrm{C}$ para que fosse avaliado o teor real de argila dos compósitos. A análise de TGA também foi empregada para calcular a porcentagem empírica de argila incorporada em cada amostra de nanocompósito. Os teores foram calculados a partir do resíduo obtido na análise de TGA, por meio de uma interpolação, como mostra a Equação 1.

$$
\frac{T A}{100}=\frac{\text { Resíduo Nanocompósito }- \text { Resíduo Polímero }}{\text { Resíduo Argila }- \text { Resíduo Polímero }}
$$


Na Equação 1, TA é o teor de argila no compósito. O teor de argila no PBS puro equivale a $0 \%$, enquanto o teor residual de argila na argila pura equivale a $100 \%$ de argila.

\section{Calorimetria exploratória diferencial (DSC)}

As propriedades térmicas, como os valores das temperaturas de transição vítrea $\left(\mathrm{T}_{\mathrm{g}}\right)$, cristalização $\left(\mathrm{T}_{\mathrm{c}}\right)$, fusão cristalina $\left(\mathrm{T}_{\mathrm{m}}\right)$ e os valores de suas cristalinidades $\left(\mathrm{X}_{\mathrm{c}}\right)$ das amostras, foram estudadas por calorimetria exploratória diferencial (DSC), feita em equipamento TA Instruments DSC Q-1000 V9.9 Build 303 na faixa de -80 a $140{ }^{\circ} \mathrm{C}$, com taxa de aquecimento de $10^{\circ} \mathrm{C} \cdot \mathrm{min}^{-1}$, com massa da amostra de aproximadamente $15 \mathrm{mg}$, sob fluxo de $\mathrm{N}_{2}\left(50 \mathrm{~mL} \cdot \mathrm{min}^{-1}\right)$. Por meio dessa análise é possível obter a cristalinidade, bem como os valores das temperaturas de transição vítrea $\left(\mathrm{T}_{\mathrm{g}}\right)$, de cristalização $\left(\mathrm{T}_{\mathrm{c}}\right)$ e de fusão cristalina $\left(\mathrm{T}_{\mathrm{m}}\right)$ do PBS e dos nanocompósitos. Para o cálculo da cristalinidade, o valor da entalpia de fusão de uma amostra $100 \%$ cristalina de PBS é igual a $110,3 \mathrm{~J} / \mathrm{g}^{[17]}$.

\section{Difração de raios $X(D R X)$}

Os ensaios de DRX foram efetuados em difratômetro da marca Rigaku, modelo Miniflex, em ângulo $2 \theta$ de $2^{\circ}$ a $50^{\circ}$, pelo método FT (tempo fixo), sob passo de $0,05^{\circ}$ por segundo. A análise foi feita em temperatura ambiente com radiação $\mathrm{K} \alpha$ de $\mathrm{Cu}(\lambda=1,5418 \AA)$. Esta técnica permite observar o pico característico das lamelas de silicato da argila (plano cristalino (001)), assim como o deslocamento, resultante do efeito da inserção da argila no polímero. $\mathrm{O}$ espaçamento interlamelar (basal) das camadas de silicato, ou seja, a distância entre as lamelas $\left(\mathrm{d}_{001}\right)$; pode ser quantificada com auxílio da Lei de Bragg.

\section{Reologia do polímero fundido}

Os ensaios reológicos das amostras de PBS e dos compósitos foram efetuados no Reômetro da TA Instruments modelo Advanced Rheometer (AR 2000), usando geometria do tipo cone-placa, de diâmetro de $25 \mathrm{~mm}$, a $120^{\circ} \mathrm{C}$ e a uma taxa de cisalhamento de $0,1 \mathrm{~s}^{-1}$ a $1000 \mathrm{~s}^{-1}$.

\section{Microscopia eletrônica de varredura (MEV)}

Para os ensaios de microscopia eletrônica de varredura, as amostras foram fraturadas em pequenos fragmentos e colocadas em um suporte com a face fraturada voltada para cima. As amostras foram recobertas com ouro. Os estudos foram feitos em um microscópio JEOL modelo JSM5610LV. As amostras foram analisadas sob uma voltagem de aceleração de $20 \mathrm{kV}$, utilizando detectores de elétrons secundários na ampliação de 1000 vezes.

\section{Ressonância magnética nuclear de baixo campo (RMN-BC)}

A análise da dinâmica molecular de um compósito é importante na investigação da qualidade da interação polímero-carga ${ }^{[18]}$. Neste trabalho foi utilizado para estudo o tempo de relaxação $T_{1}$ do núcleo de hidrogênio $\left(T_{1} H\right)$, com o objetivo de avaliar a dispersão da carga, a argila, na matriz polimérica de PBS. Para a determinação das medidas de relaxação nuclear, foi utilizado um espectrômetro de ressonância magnética nuclear de baixo campo da Oxford Instruments, modelo Maran Ultra 23, operando na frequência de 23,4 MHz. O tempo de relaxação spin-rede do hidrogênio foi determinado diretamente pela sequência de pulso inversão-recuperação. A temperatura empregada foi de $27^{\circ} \mathrm{C}$. A faixa de tau utilizada $(\tau)$ foi de $0,1-5000$ milissegundos (ms), com 4 medidas para cada ponto, com um intervalo de reciclo de $1 \mathrm{~s}$.

\section{Resultados e Discussão}

\section{FTIR-ATR}

Os espectros do PBS puro, dos compósitos e da argila organofílica são mostrados na Figura 1.

As bandas que aparecem em 1330 e 2945 cm$^{-1}$ são atribuídas às vibrações simétricas e assimétricas de deformação de grupos $-\mathrm{CH}_{2}$ - na cadeia principal do PBS, respectivamente. Já bandas em torno de 1144 e $1264 \mathrm{~cm}^{-1}$ correspondem ao estiramento das ligações -C-O-C- no grupo éster de PBS. A banda em $1714 \mathrm{~cm}^{-1}$ é referente às vibrações de alongamento do grupo éster no PBS, mais especificamente nas ligações $\mathrm{C}=\mathrm{O}$ da carbonila. As bandas na região de $1044 \mathrm{~cm}^{-1}$ foram relacionadas às vibrações de alongamento das ligações O-C-C no $\mathrm{PBS}^{[19]}$. A banda em torno de $3453 \mathrm{~cm}^{-1}$ pode ser atribuída ao grupo terminal hidroxila do $\mathrm{PBS}^{[20]}$. Também é possível visualizar bandas na região de $914 \mathrm{~cm}^{-1}$ que corresponde a grupos vinil. A ocorrência de grupos terminais vinil é atribuída à reação de cisão de cadeia do $\mathrm{PBS}^{[21]}$.

Como os espectros dos compósitos apresentam somente as bandas características do PBS, na tentativa de constatar a incorporação da argila no polímero, foi feito um tratamento estatístico a partir desses espectros, comparando-os. Para isso, foi usada uma ferramenta estatística, a técnica dos mínimos quadrados, que permite calcular a correlação e o erro quadrático médio (RMSE) entre a população em estudo (valores experimentais) e o modelo. O RMSE, ou raiz do erro quadrático médio, é capaz de medir a dispersão entre esses valores ${ }^{[22]}$. Assim, foi avaliada a diferença entre os espectros dos compósitos e o do PBS puro. A Figura 1 também mostra esses gráficos comparativos. Os valores de RMSE entre os pares PBS/PBS; PBS / PBS-4; PBS / PBS6; e PBS / PBS-8 foram iguais a 0,$0000 ; 1,3840 ; 3,0584$; e 3,3028 , respectivamente. Esses resultados provam que a adição da argila na matriz polimérica produz interferência nos espectros, aumentando linearmente $\left(\mathrm{R}^{2}=0,95\right)$ os valores de RMSE dos compósitos com o aumento da quantidade de argila usada. Portanto, o teste de RMSE constitui um excelente indicativo da presença da fase inorgânica dispersa na matriz polimérica.

\section{Difração de raios $X(D R X)$}

Os difratogramas da argila organofílica e dos compósitos PBS-4, PBS-6 e PBS-8 são mostrados na Figura 2, onde observa-se que nas amostras PBS-4 e PBS-8 ocorreu o deslocamento do pico $2 \theta$ correspondente ao plano cristalino (001) de 4,6 $\left(\mathrm{d}_{001}=19,19 \AA\right)$ para, aproximadamente, $2,6^{\circ}$ $\left(\mathrm{d}_{001}=33,93 \AA\right)$.

Isso sugere a formação de uma estrutura em que as cadeias de PBS estão intercaladas entre as lamelas da argila. No entanto, estes picos não estão muito bem definidos, o que pode ser sinal de que ocorreu certo grau de esfoliação da argila na matriz do polímero. Esse deslocamento ocorre, 

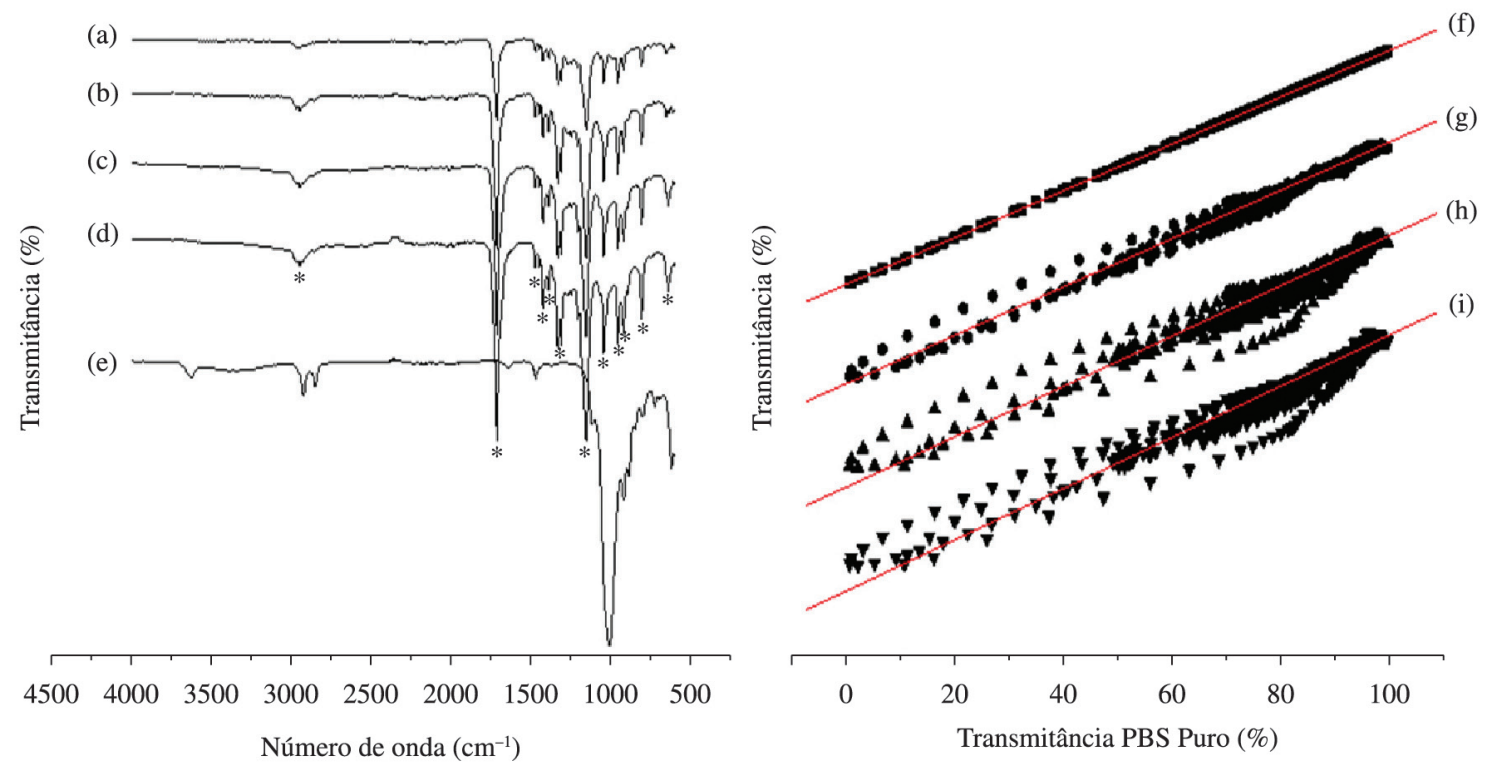

Figura 1. A esquerda, espectros de infravermelho do PBS puro (a); dos compósitos contendo 4\% (b), 6\% (c) e 8\% (d) de argila; e da argila organofílica (e). À direita, análise comparativa dos valores de transmitância do PBS (f) e dos compósitos contendo 4\% (g), 6\% (h) e $8 \%$ (i) de argila versus a transmitância do PBS usados para os cálculos de RMSE.

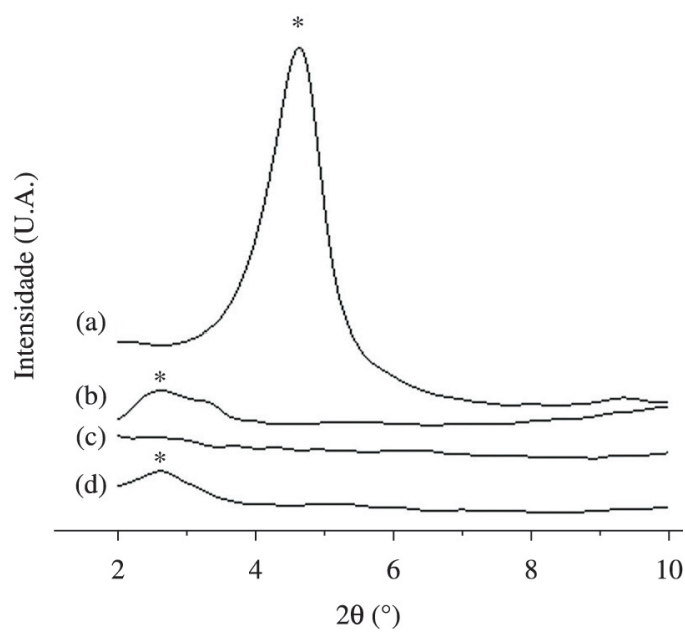

Figura 2. Difratograma da montmorilonita organofílica (a) e dos compósitos contendo $4 \%$ (b), $6 \%$ (c) e $8 \%$ (d) de argila. *Pico de difração atribuído à argila.

pois o crescimento das cadeias no interior dessas camadas durante a polimerização in situ faz com que haja um maior afastamento entre as lamelas, aumentando a distância entre elas (espaçamento basal). A amostra PBS-6, no entanto, não apresentou o pico do plano (001). Uma explicação a ser considerada é a esfoliação das lamelas da argila na matriz polimérica, devido a uma melhor dispersão da carga. Neste caso as lamelas da argila estão tão bem dispersas na matriz que já não se faz presente a estrutura de camadas. Logo, por DRX, não é possível calcular a distância entre as lamelas ${ }^{[23]}$. A literatura demonstra que a obtenção desses sistemas via fusão é passível de resultados divergentes. Por exemplo, entre os estudos disponíveis, o trabalho de Phua et al. ${ }^{[24]}$ destaca que nanocompósitos de PBS/argila foram obtidos por intercalação no polímero fundido, com 2 e $4 \%$ de argila montmorilonita; sendo assim, seus difratogramas não apresentaram o pico característico das lamelas de silicato. Amostras com teores mais elevados (5 e 10\%) apresentaram, em seus difratogramas, o pico correspondente ao espaçamento interlamelas das argilas, indicando a obtenção da estrutura esfoliada. Por sua vez, o trabalho de Ray et al. ${ }^{[25]}$ demonstrou que amostras de nanocompósitos de PBS/argila montmorilonita, também sintetizados por fusão, com teores de 3 e $5 \%$ de argila, apresentaram o pico de difração das lamelas de silicato da argila. Desta forma, fica demonstrado que o método de preparo via fusão, mesmo com baixos teores de argila, também pode produzir tanto compósitos de estrutura esfoliada quanto intercalada.

As análises de DRX permitiram inferir que ocorreu a incorporação da argila no PBS, assim como a presença de pelo menos duas estruturas do compósito: a intercalada e a esfoliada. Como as lamelas de silicato possuem a espessura de cerca de $1 \mathrm{~nm}$, as estruturas formadas podem ser consideradas estruturas nanométricas.

\section{Reologia do nanocompósito}

A resposta para a reologia sob cisalhamento contínuo dos nanocompósitos é apresentada na Figura 3.

Comparando os resultados dos ensaios de reologia com cisalhamento contínuo dos nanocompósitos e do PBS puro nota-se que houve a redução da viscosidade com a adição da argila na matriz polimérica via polimerização in situ. O PBS e os nanocompósitos contendo 4,6 e $8 \%$ de argila apresentaram viscosidades médias iguais a $4,54 \pm 0,60$, $0,68 \pm 0,04,0,29 \pm 0,02$ e $0,23 \pm 0,02$ Pa.s, respectivamente. A possível causa dessa redução é a dificuldade de acesso 


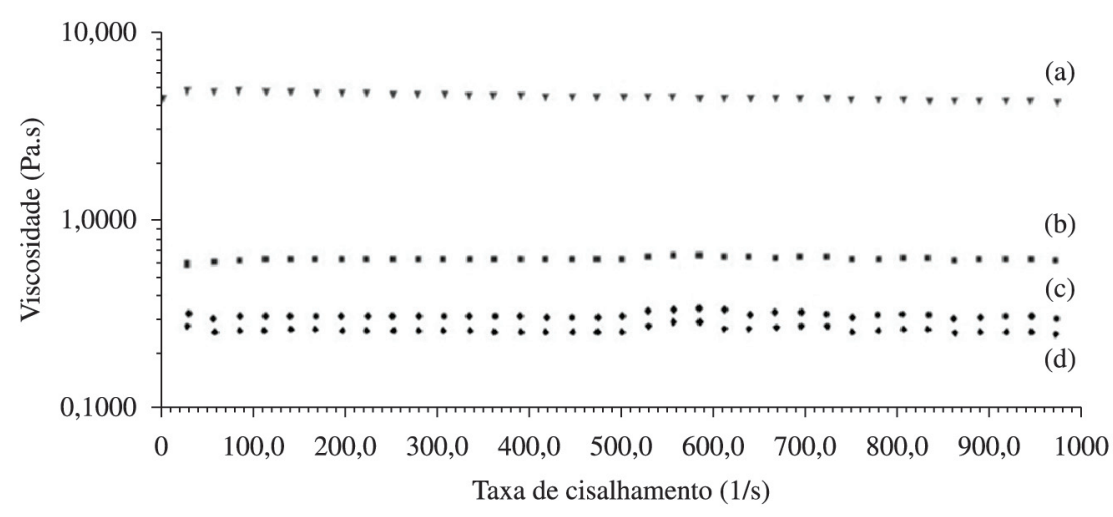

Figura 3. Resposta ao cisalhamento contínuo do PBS (a) e dos compósitos contendo 4\% (b), 6\% (c) e $8 \%$ (d) de argila.

dos monômeros aos centros ativos do catalisador devido à presença da argila no meio desde o início da reação, o que pode ter inibido o crescimento das cadeias do polímero e contribuído para a obtenção do PBS com menor massa molar, o que contribui para a redução da viscosidade dos nanocompósitos.

\section{Análise térmica}

Os resultados de TGA são mostrados na Figura 4 e na Tabela 1.

A estrutura lamelar das argilas tem a capacidade de melhorar as propriedades térmicas dos polímeros devido aos efeitos de barreira, que dificultam a difusão dos produtos voláteis da decomposição, resultado da redução da permeabilidade observada em nanocompósitos com estrutura esfoliada ${ }^{[26]}$. No trabalho desenvolvido por Bathia e colaboradores (2009), foi observado o aumento da estabilidade térmica da blenda polimérica de PLA/ PBS (poli(ácido láctico)/ poli(succinato de butileno)) com adição de argila montmorilonita para teores acima de 3\%. Esse efeito foi demonstrado pelo aumento da temperatura inicial de degradação (onset) com a adição da argila na matriz polimérica ${ }^{[27]}$.

No entanto, com o aumento do teor de argila, foi observada uma tendência de redução da estabilidade através da redução dos valores de temperatura de máxima taxa de degradação de $422^{\circ} \mathrm{C}$ (PBS puro) para $398^{\circ} \mathrm{C}$ (PBS-8). Este fato revela que a redução da massa molar do PBS, verificada pela diminuição da viscosidade, causou um efeito muito maior sobre a resistência térmica do polímero, comparado ao efeito da carga na matriz polimérica.

O cálculo dos teores de argila dos nanocompósitos foi feito a partir de uma interpolação dos valores dos resíduos da análise termogravimétrica. Os teores calculados (ver Tabela 1) permitem inferir que os valores de concentração reais de argila em cada amostra são próximos aos valores teóricos, apresentando uma diferença relativa acumulada igual a $13 \pm 4 \%$.

Por sua vez, os resultados de DSC mostram que a redução da massa molar também afetou as propriedades térmicas, causando a diminuição das temperaturas de transição vítrea $\left(\mathrm{T}_{\mathrm{g}}\right)$, cristalização $\left(\mathrm{T}_{\mathrm{c}}\right)$ e fusão cristalina $\left(\mathrm{T}_{\mathrm{m}}\right)$ do PBS, uma vez considerada a relação direta existente

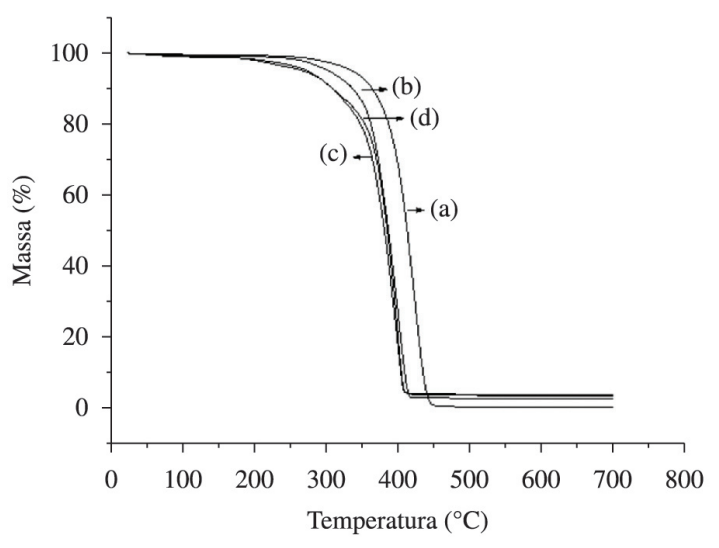

Figura 4. Curvas termogravimétricas do PBS puro (a) e dos compósitos contendo 4\% (b), 6\% (c) e 8\% (d) de argila.

entre as propriedades térmicas de um polímero com a sua massa molar ${ }^{[28]}$. Em relação à cristalinidade, a partir do teor de cerca de $4 \%$, a incorporação da argila no PBS afeta a formação dos cristais, pois dificulta a ordenação das cadeias poliméricas. Logo, com o aumento do teor de argila há uma tendência de redução da cristalinidade.

\section{Microscopia eletrônica de varredura (MEV)}

A morfologia das amostras de PBS e PBS/argila foi avaliada por MEV. A Figura 5 mostra as micrografias das amostras analisadas. Como é possível notar, existem diferenças na morfologia das amostras, dependendo do teor de argila.

Todas as amostras apresentam uma matriz polimérica fraturada, devido à ruptura executada manualmente; porém, com o aumento do teor de argila, ocorre o surgimento e gradual aumento da concentração de aglomerados pontuais. Seguindo essa tendência, percebe-se nas fotomicrografias que a amostra PBS-4 não apresenta aglomerados visíveis, enquanto na amostra PBS-6 já é possível observar a formação de aglomerados. A amostra PBS-8, por sua vez, exibe uma particularidade: a formação de "vazios" na matriz polimérica. Esse fenômeno é notavelmente causado pela formação de aglomerados ainda maiores, que se localizam 
Tabela 1. Teores de argila, propriedades térmicas e cristalinidade do PBS e dos nanocompósitos.

\begin{tabular}{lcccccc}
\hline \multicolumn{1}{c}{ Amostra } & $\mathbf{T}_{\mathbf{d}}\left({ }^{\circ} \mathbf{C}\right)^{*}$ & Argila $(\boldsymbol{\%}) \#$ & $\mathbf{T}_{\mathbf{g}}\left({ }^{\mathbf{C}} \mathbf{C}\right)$ & $\mathbf{T}_{\mathbf{c}}\left({ }^{\circ} \mathbf{C}\right)$ & $\mathbf{T}_{\mathbf{m}}\left({ }^{\circ} \mathbf{C}\right)$ & $\mathbf{X}_{\mathbf{c}}(\boldsymbol{\%})$ \\
\hline PBS & 422 & 0,00 & -28 & 80 & 114 & 71 \\
PBS-4 & 400 & 3,54 & -30 & 73 & 110 & 71 \\
PBS-6 & 398 & 4,93 & -39 & 66 & 104 & 60 \\
PBS-8 & 398 & 7,22 & -35 & 69 & 105 & 57 \\
Argila Organofílica & - & 100 & - & - & - & - \\
\hline
\end{tabular}

\#Temperatura tomada na taxa máxima de degradação; *Teor calculado por TGA.
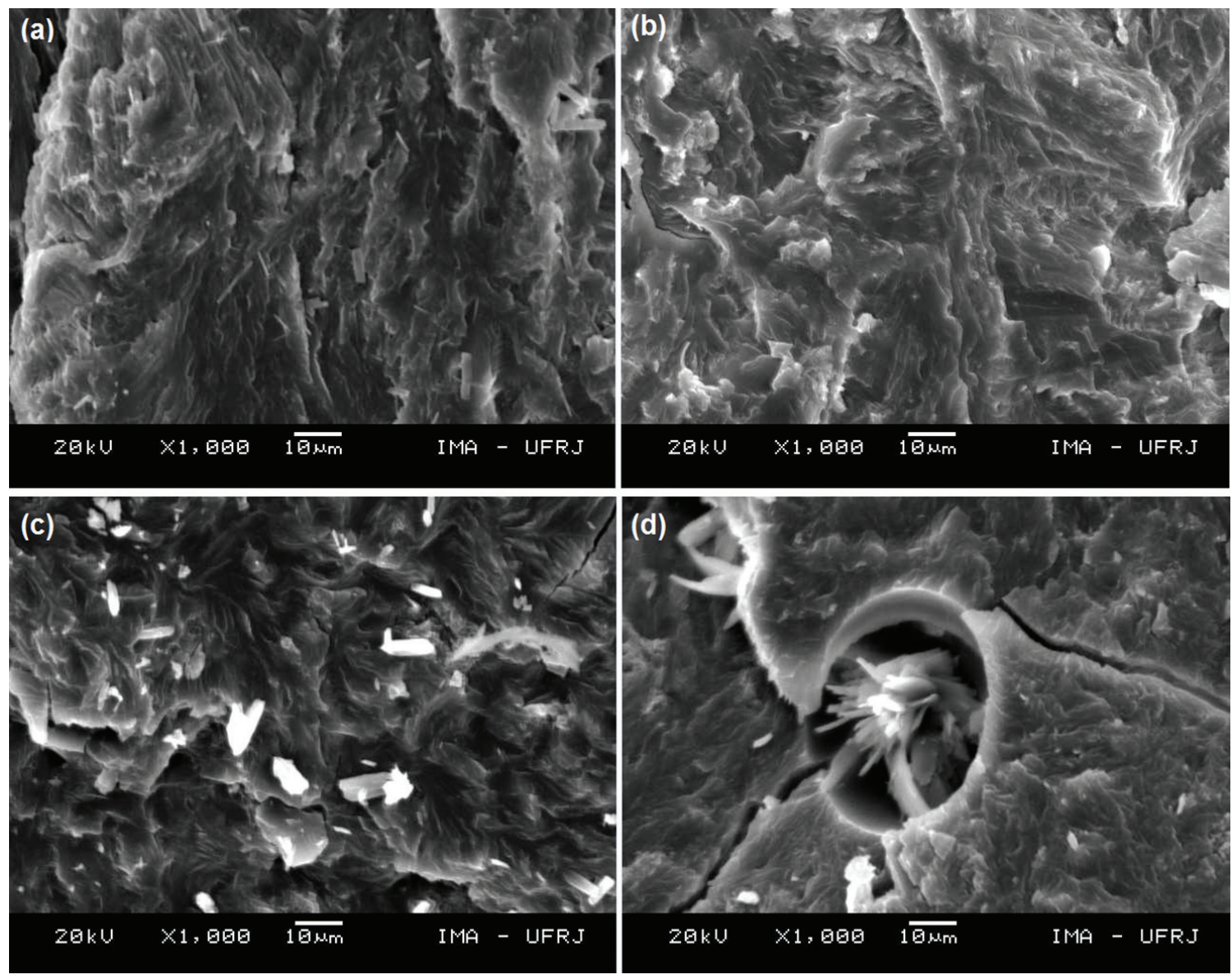

Figura 5. Fotomicrografias do PBS (a) e dos compósitos contendo 4\% (b), 6\% (c) e 8\% (d) de argila.

no interior destes vazios. Desta forma, pode-se verificar que menores quantidades de argila acarretam uma melhor dispersão da carga e que quantidades elevadas de carga produzem uma maior formação desses aglomerados, devido a uma pior dispersão da mesma.

Como esta técnica só permite a observação das frações compósitas das amostras onde estão presentes aglomerados de argila, se torna relevante o emprego de outras técnicas para a investigação, como por exemplo, a técnica de ressonância magnética nuclear de baixo campo.

\section{RMN de baixo campo (RMN-BC)}

A técnica RMN-BC constitui uma interessante forma de caracterização de materiais poliméricos. Os valores dos tempos de relaxação $T_{1}$ permitem avaliar a mobilidade molecular das cadeias, umas em relação às outras. Dessa forma, é possível fazer distinção entre átomos de hidrogênio que estão em domínios de mobilidades distintas. Como exemplo, podem ser identificados os átomos de hidrogênio das cadeias poliméricas que estão intercaladas, os átomos de hidrogênio das cadeias poliméricas que estão em torno das lamelas da argila e os que estão em torno de um aglomerado de argila ${ }^{[29]}$. Assim, a técnica RMN-BC possibilitou observar o efeito da carga sobre a relaxação do PBS. A Figura 6 exibe a evolução da dinâmica molecular do polímero, da argila e dos nanocompósitos a partir dos valores de $\mathrm{T}_{1} \mathrm{H}$ dos átomos de hidrogênio presentes nos diferentes domínios dos materiais, representados nas curvas resposta.

A curva referente à argila apresenta domínios em valores menores de $\mathrm{T}_{1} \mathrm{H}$, em torno de 0,5-1 ms. Isso 


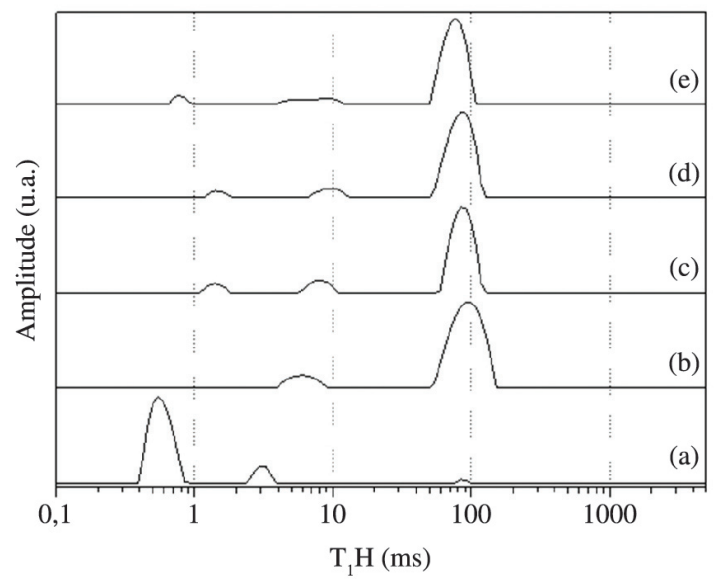

Figura 6. Curva de distribuição de domínios de $\mathrm{T}_{1} \mathrm{H}$ para a montmorilonita organofílica (a); para o PBS (b) e para os compósitos contendo 4\% (c), 6\% (d) e 8\% (e) de argila.

ocorre porque os átomos de ${ }^{1} \mathrm{H}$ contidos na argila possuem uma alta mobilidade, tanto na estrutura octaédrica da argila quanto nas cadeias hidrocarbônicas dos cátions de sais quaternários presentes na região interlamelar. Além disso, o paramagnetismo dos íons metálicos existentes na constituição das lamelas $\left(\mathrm{Fe}^{3+}\right)$ causa um decréscimo no valor do tempo de relaxação do núcleo de hidrogênio. Já a curva correspondente ao PBS puro apresenta domínios em valores maiores de $\mathrm{T}_{1} \mathrm{H}$, em torno de $100 \mathrm{~ms}$, pois os átomos de hidrogênio pertencentes às cadeias poliméricas não possuem tanta mobilidade, devido à conformação emaranhada das longas cadeias do polímero e ao elevado contato entre elas ${ }^{[30]}$. Dessa forma os átomos de ${ }^{1} \mathrm{H}$ contidos na cadeia polimérica apresentam maiores tempos de relaxação. É possível observar que esta curva apresenta picos referentes a dois domínios: um pequeno, para valores menores de $\mathrm{T}_{1} \mathrm{H}$ (5-10 ms), e um maior, em valores maiores de $\mathrm{T}_{1} \mathrm{H}(40-110 \mathrm{~ms})$. Esse fato é explicado pela conformação do polímero. O PBS, polímero bastante cristalino, apresenta um domínio predominante (pico de maior área) correspondente aos átomos de hidrogênio contidos nas moléculas ordenadas em uma estrutura cristalina, e que, portanto, possuem menor mobilidade e tempos de relaxação maiores. Já o domínio localizado em valores menores de $\mathrm{T}_{1} \mathrm{H}$ refere-se aos átomos de hidrogênio das cadeias poliméricas presentes nas regiões amorfas, sendo assim mais livres, o que resulta no menor tempo de retorno ao estado fundamental ${ }^{[31]}$. Por sua vez, os nanocompósitos apresentam um domínio menos significativo, representado em menores valores de $\mathrm{T}_{1} \mathrm{H}(0,5-2 \mathrm{~ms})$, que corresponde a pequenos aglomerados de argila presentes nos nanocompósitos. Ainda analisando as curvas dos nanocompósitos, observa-se que o pico correspondente ao domínio amorfo tem um leve deslocamento para maiores valores de $\mathrm{T}_{1} \mathrm{H}$, de $5 \mathrm{~ms}$ para aproximadamente $10 \mathrm{~ms}$, o que sugere um ganho de rigidez da estrutura; ou seja, a perda de mobilidade adquirida pelas cadeias que constituem estas regiões ${ }^{[32]}$. Essa perda de mobilidade pode ser justificada pela boa interação entre as cadeias do polímero e a argila organofílica. Há também uma tendência à redução dos valores de $\mathrm{T}_{1} \mathrm{H}$ dos átomos de hidrogênio presentes na região cristalina, cujo pico se localiza na região de 80-100 ms. Esse deslocamento do pico correspondente aos domínios está diretamente relacionado ao efeito da argila na matriz polimérica e com o rearranjo da estrutura original do polímero. Quanto mais dispersa a argila estiver no meio, caracterizando uma estrutura esfoliada, maior mobilidade terão as cadeias do polímero, devido ao menor contato destas entre si e do maior contato destas com a argila ${ }^{[33]}$. A presença de cadeias poliméricas em torno das lamelas da argila também faz com que os núcleos de hidrogênio estejam mais próximos aos metais paramagnéticos, reduzindo os valores de $\mathrm{T}_{1} \mathrm{H}$. Deste modo, pode-se inferir sobre a estrutura dos nanocompósitos por meio do deslocamento dos valores de $\mathrm{T}_{1} \mathrm{H}$ que remetem aos domínios. Neste caso, houve a redução dos valores de $\mathrm{T}_{1} \mathrm{H}$, o que indica a predominância de uma estrutura esfoliada nos nanocompósitos ${ }^{[34]}$. Esse resultado agrega significado aos resultados obtidos pela técnica de difração de raios X, indicando que os picos pouco definidos observados nos difratogramas, referentes à argila, caracterizam a formação de uma estrutura esfoliada, predominantemente. Além disso, o pico correspondente ao domínio cristalino sofreu um estreitamento, o que sugere a formação de cristais mais imperfeitos e demonstra o efeito negativo da incorporação da argila sobre a cristalinidade ${ }^{[35]}$.

\section{Conclusões}

A síntese dos compósitos via polimerização in situ promoveu a polimerização em meio às lamelas da argila, o que levou a formação de compósitos com estruturas esfoliadas, intercaladas, além dos tactóides (aglomerados). Estas estruturas foram confirmadas pelas técnicas de difração de raios $\mathrm{X}$ e ressonância magnética nuclear de baixo campo. Esta última indicou a predominância da estrutura esfoliada, como esperado pela técnica de inserção escolhida. Por outro lado, a caracterização dos nanocompósitos por reometria mostrou que a presença de argila dificultou o crescimento das cadeias, promovendo a redução da estabilidade térmica e da temperatura de fusão cristalina dos materiais. A presença de aglomerados na matriz polimérica também pode ter contribuído para esses resultados. Assim, esse trabalho demonstrou a obtenção de nanocompósitos de PBS argila por meio da técnica de polimerização in situ, feita em massa. Mais que isso, esse artigo contribuiu para o avanço do conhecimento sobre o preparo de materiais nanocompósitos de matriz PBS. Esse polímero de excelentes propriedades, devido ao barateamento da matéria prima obtido por rota biotecnológica, será, nos próximos anos, um material plataforma para a indústria polimérica. Essa realidade próxima deve servir como um incentivo à comunidade no sentido de novas pesquisas relacionadas a sua síntese, caracterização e aplicações, cada vez mais relevantes.

\section{Agradecimentos}

Os autores agradecem ao CNPq, à CAPES e CAPESNANOBIOTEC, à FINEP (ref.1889/10) e à FAPERJ pelo apoio financeiro e pelas bolsas. 


\section{Referências Bibliográficas}

1. Jacquel, N.; Freyermouth, F.; Fenouillot, F.; Rousseau, A.; Pascault, J. P.; Fuertes, P. \& Saint-Loup, R.-J. - J. Polym. Sci. A Polym. Chem., 49, p.5301 (2011).

2. Wang, G.; Gao, B.; Ye, H.; Xu, J. \& Guo, B.-J. - J. Appl. Polym. Sci., 117, p.2538 (2010).

3. Andrade, F. D.; Gonzales, T.; Alegre, R. M. \& Duarte, E. R. Evidência, 10, p.27 (2010).

4. Tan, L.; Chen, Y.; Zhou, W.; Nie, H.; Li, F. \& He, X. - Polym. Degrad. Stabil., 95, p.1920 (2010). http://dx.doi.org/10.1016/j. polymdegradstab.2010.04.010.

5. Vassiliou, A. A.; Bikiaris, D.; El Mabrouk, K. \& Kontopoulou, M. - J. Appl. Polym. Sci., 119, p.2010 (2011). http://dx.doi. org/10.1002/app.32887.

6. Nguyen, Q. T. \& Baird, D. G. - Adv. Polym. Technol., 25, p.270 (2006). http://dx.doi.org/10.1002/adv.20079.

7. Sinha Ray, S. \& Okamoto, M. - Prog. Polym. Sci., 28, p.1539 (2003). http://dx.doi.org/10.1016/j.progpolymsci.2003.08.002.

8. Chen, Y.; Zhan, J.; Zhang, P.; Nie, S.; Lu, H.; Song, L. \& Hu, Y. - Ind. Eng. Chem. Res., 49, p.8200 (2010). http://dx.doi. org/10.1021/ie100989j.

9. Alexandre, M. \& Dubois, P. - Mater. Sci. Eng., 28, p.1 (2000). http://dx.doi.org/10.1016/S0927-796X(00)00012-7.

10. Scocchi, G.; Posocco, P.; Fermeglia, M. \& Pricl, S. - J. Phys. Chem. B., 111, p.2143 (2007).

11. Paiva, L. B.; Morales, A. R. \& Díaz, F. R. V. - Ceramica., 54, p.213 (2008). http://dx.doi.org/10.1590/S036669132008000200012.

12. Phua, Y. J.; Chow, W. S. \& Mohd Ishak, Z. A. - Polym. Degrad. Stabil., 96, p.1194 (2011). http://dx.doi.org/10.1016/j. polymdegradstab.2011.04.017.

13. Ray, S. S. \& Bousmina, M. - Polymer, 46, p.12430 (2005). http://dx.doi.org/10.1016/j.polymer.2005.10.102.

14. Carastan, D. J. \& Demarquette, N. R. - Int. Mater. Rev., 52, p.345 (2007). http://dx.doi.org/10.1179/174328007X212517.

15. Chaeichian, S.; Wood-Adams, P. M. \& Hoa, S. V. Polymer, 54, p.1512 (2013). http://dx.doi.org/10.1016/j. polymer.2012.09.021.

16. Ferreira, L. P.; Moreira, A. N.; Delazare, T.; Oliveira, G. E. \& Souza, F. G. Jr - Macromol. Symp., 319, p.210 (2012). http:// dx.doi.org/10.1002/masy.201100145.

17. Xu, Y.; Xu, J.; Liu, D.; Guo, B. \& Xie, X.-J. - Appl. Polym. Sci., 109, p.1881 (2008). http://dx.doi.org/10.1002/app.24544.

18. Rodrigues, E. J. R.; Nascimento, S. A. M.; Tavares, M. I. B. \& Merat, P. P. - Polímeros, 22, p.436 (2012). http://dx.doi. org/10.1590/S0104-14282012005000058.
19. Kim, H. S.; Kim, H. J.; Lee, J. W. \& Choi, I.-G. - Polym. Degrad. Stabil., 91, p.1117 (2006). http://dx.doi.org/10.1016/j. polymdegradstab.2005.07.002.

20. Chen, C. H.; Peng, J. S.; Chen, M.; Lu, H. Y.; Tsai, C. J. \& Yang, C. S. - Colloid Polym. Sci., 288, p.731 (2010). http:// dx.doi.org/10.1007/s00396-010-2187-9.

21. Ahmad Thirmizir, M. Z.; Mohd Ishak, Z. A.; Mat Taib, R.; Rahim, S. \& Mohamad Jani, S. - J. Polym. Environ., 19, p.263 (2011). http://dx.doi.org/10.1007/s10924-010-0272-2.

22. Kelley, K. \& Lai, K. - Multivariate Behav. Res., 46, p.1 (2011). http://dx.doi.org/10.1080/00273171.2011.543027.

23. Sinha Ray, S.; Okamoto, K. \& Okamoto, M. - Macromolecules., 36, p.2355 (2003). http://dx.doi.org/10.1021/ma021728y.

24. Phua, Y. J.; Chow, W. S. \& Ishak, Z. A. M. - J. Thermoplast. Comps., 24, p.133 (2011).

25. Ray, S. S.; Okamoto, K.; Maiti, P. \& Okamoto, M. - J. Nanosci. Nanotechno., 2, p.171 (2002). http://dx.doi.org/10.1166/ jnn.2002.086.

26. Mignoni, M. L.; Silva, J. V. M.; Souza, M. O.; Mauler, R. S.; Souza, R. F. \& Gusmão, K. B. - J. Appl. Polym. Sci., 122, p.2159 (2011)

27. Bhatia, A.; Gupta, R. K.; Bhattacharya, S. N. \& Choi, H. J. - J. Appl. Polym. Sci., 114, p.2837 (2009). http://dx.doi. org/10.1002/app.30933

28. Canevarolo, S. V. Jr - "Ciência dos polímeros: um texto básico para tecnólogos e engenheiros" 2 nd ed., Artliber, São Paulo (2006).

29. Silva, M. A.; Tavares, M. I. B.; Nascimento, S. A. M. \& Rodrigues, E. J. R. - Polímeros, 22, p.481 (2012). http://dx.doi. org/10.1590/S0104-14282012005000064.

30. Rodrigues, E. J. R.; Nascimento, S. A. M.; Tavares, M. I. B. \& Merat, P. P. - Polímeros, 22, p.436 (2012). http://dx.doi. org/10.1590/S0104-14282012005000058.

31. Resende, D. K.; Dornelas, C. B.; Tavares, M. I. B.; Gomes, A. S.; Moreira, L. A.; Cabral, L. M. \& Simeoni, L. A. Polímeros, 20, p.231 (2010). http://dx.doi.org/10.1590/ S0104-14282010005000031.

32. Rodrigues, T. C.; Tavares, M. I. B.; Preto, M.; Soares, I. L. \& Moreira, A. C. F. - Int. J. Polym. Mater., 57, p.1119 (2008).

33. Merat, P. P.; Tavares, M. I. B. \& Silva, E. O. - J. Mater. Sci. Eng. A, 1, p.671 (2011).

34. Silva, P. S. R. C. - Polímeros, 23, p.644 (2013).

35. Silva, M. B. R.; Tavares, M. I. B.; Silva, E. O. \& Neto, R. P. C. - Polym. Test., 32, p.165 (2013). http://dx.doi.org/10.1016/j. polymertesting.2012.09.006.

Enviado: Fev. 09, 2014

Reenviado: Abr. 15, 2014

Aceito: Abr. 24, 2014 